\title{
Robust Dynamic Modeling and Control of Dual-Stage Actuators
}

\author{
Raymond A. de Callafon ${ }^{1}$, Ryozo Nagamune ${ }^{2}$, and Roberto Horowitz ${ }^{2}$ \\ ${ }^{1}$ Department of Mechanical and Aerospace Engineering, University of California at San Diego, La Jolla, CA 92093 USA \\ ${ }^{2}$ Department of Mechanical Engineering, University of California at Berkeley, Berkeley, CA 94720 USA
}

\begin{abstract}
In a hard disk drive with a dual-stage actuator the standard rotary actuation of the voice coil motor (VCM) is combined with an additional micro- or milli-actuation to accomplish high-bandwidth and highly accurate track following. For extremely high density recording, the servo control algorithm designed for a specific dual-stage actuator needs to maintain high performance track following in lieu of external disturbances and uncertainties in the dynamic response induced by product manufacturing tolerances. Essential in the robust design of a servo control algorithm is to bound these uncertainties and design a robust track following servos accordingly. In this paper, we present an experiment based methodology to characterize and bound the uncertainties in the dynamic response of a dual-stage actuator in the form of an uncertainty model. Subsequently, the uncertainty model is used for the analysis and synthesis of a robust dual-stage servo controller that is able to optimize position error variance in the presence of uncertainty information.
\end{abstract}

Index Terms-Dual-stage actuator, robust control, servo control, uncertainty.

\section{INTRODUCTION}

$\mathbf{S}$ IGNIFICANT progress in areal storage density of a magnetic hard disk drive (HDD) can be accomplished by using a dual-stage actuator system. In such a servo system a high-bandwidth and highly accurate micro-actuator (MA) is used in combination with a traditional voice coil motor (VCM) to position the read/write head on the slider over the data track [1]-[4]. While most high density demonstrations are achieved under well-conditioned laboratory environments, the servo system in a commercial HDD is subjected to inconsistencies due to manufacturing tolerances. If a high storage areal density needs to be achieved in a low cost consumer application, then the track following servo control system is required to perform robustly in the presence of uncertainties induced by disturbances and product variability.

Via modern control design approaches that make use of $H_{\infty}$ and $\mu$ synthesis [5]-[7] it is possible to incorporate the effects of unknown disturbances and product variations. For the design of a robust servo system, uncertainties are taken into account in the form of an uncertainty model. The uncertainty model consists of a nominal model together with a description of the perturbation of the nominal model and an unknown but bounded description of the disturbances and uncertainty.

This paper presents a methodology for obtaining a robust dual-stage servo control system. The methodology consists of characterizing both structured and nonstructured uncertainty models on the basis of experimental data obtained form several dual-stage actuators. On the basis of the uncertainty model subsequently a robust controller design technique is used to design a robustly performing dual-stage servo controller. The methodology is illustrated on a dual-stage servo system with a PZT-actuated suspension.

Digital Object Identifier 10.1109/TMAG.2005.861738

\section{UNCERTAINTY MODEL CHARACTERIZATION}

\section{A. PZT-Actuated Suspension}

The experimental results presented in this paper are based on a dual-stage servo system that utilizes PZT-actuated suspensions manufactured by Hutchinson as the second-stage actuator. In the HIT Magnum 5E PZT-actuated suspension, two PZT bars are used to move the slider in a direction perpendicular to the track by a push-pull configuration. In a hard disk with multiple disks, several suspensions are mounted on a single E-block, each carrying on its gimbal a slider with the read-write head. The E-block connects the suspensions to a radial voice coil motor (VCM) for the gross movements of all of the read/write heads. The PZT-actuated suspensions are used as a secondary actuator for the fine movements of each of the read/write heads. Generally, the servo system performs track-following control of a single read-write head at the time.

To perform the uncertainty modeling of the dual-stage servo system, experiments were conducted to observe variations in its dynamic behavior, when several PZT-actuated suspensions were installed on the E-block. The variations are primarily caused by manufacturing and E-block mounting variations of the different suspensions. Both conditions result in (uncertain) variations in the dynamic response of the dual-stage actuators, even when the PZT-suspensions are mounted on the same E-block. As multiple suspension are controlled by a single servo controller, these uncertainties have to be taken into account when designing a high-performance robust dual-stage servo controller for track following.

The variations in the dynamical behavior of the dual-stage actuator were examined by measuring the frequency response of several units. To generate experimental data, several E-blocks with four suspension connection points and nine different suspensions flying at a fixed Z-height of 22 mil were used to measure 36 frequency responses. The slider position was measured by the LDV and fed back to the DSP on which a simple proportional derivative control algorithm was implemented to stabilize the VCM and slider position for the LDV measurements. 


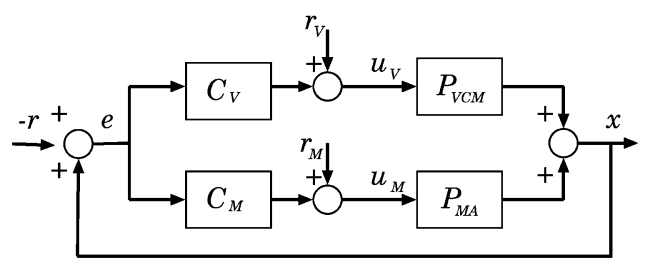

Fig. 1. Overview of signals in closed-loop dual-stage system during servo experiments.

\section{B. Identification Experiments}

Fig. 1 depicts the block diagram of the setup used to conduct the identification and servo experiments reported in this paper. The setup includes a spin stand with a 7200 RPM drive, a Polytech LDV position measurement system, which is used to measure the absolute radial position of the slider, an ST Microelectronics piezo driver, which is used to drive the PZT-actuated suspension, and a DSP analysis system, which included a Texas Instrument TMS320C6713 development DSP board and a TI floating point DSP. In Fig. 1, $P_{\mathrm{MA}}$ denotes the transfer function from the MA input signal, $u_{M}$, to the slider position $x$ as measured by the LDV, while $P_{\mathrm{VCM}}$ denotes the transfer function from the VCM input signal, $u_{V}$, to $x$. The three auxiliary excitation signals were generated by the DSP. Signal $-r$, which simulates track runout during the servo experiments reported in Section VII, is added to the slider position signal $x$ to generate the PES signal $e$, which is in turn fed to the MA and VCM compensators $C_{M}$ and $C_{V}$. Signals $r_{V}$ and $r_{M}$ are used in the identification experiments.

During the identification experiments, the runout signal, $r$, and the MA compensator, $C_{M}$, are both set to zero, while a simple proportional derivative control algorithm is implemented in $C_{V}$, to stabilize the VCM and slider position for the LDV measurements. In addition, the DSP generates two normally distributed excitation signals $r_{M}$ and $r_{V}$ of prescribed intensity in two independent experiments. As indicated in the block diagram of Fig. 1, $r_{M}$ is used to excite the MA via an ST Microelectronics piezo driver and applied to the piezo pads on the PZT-actuated suspension, while the signal $r_{V}$ is used to excite the VCM control loop. With measurements of the LDV signal $e$ and the VCM control signal $u_{V}$, estimates of the frequency response of the MA transfer function $P_{\mathrm{MA}}$ and the VCM transfer function $P_{\mathrm{VCM}}$ can be obtained via spectral analysis.

Let the notation $\hat{\Phi}_{y z}$ denote the cross-spectral estimate

$$
\hat{\Phi}_{y z} \omega=\int_{-\pi}^{\pi} W_{\gamma}(\xi-\omega) Y(\xi) d \xi
$$

where $W_{\gamma}$ is a conventional Hanning frequency window for additional averaging, and $Y(\xi), Z(\xi)$ are the $N$-point Fourier transform of the signals $y(t), z(t), t=l, 2, \ldots, N$. With the signals defined in block diagram of Fig. 1 and setting $r=C_{M}=0$, it can be verified that the frequency response of $P_{\mathrm{VCM}}$ can be estimated via

$$
\hat{P}_{\mathrm{VCM}}(\omega)=\frac{\hat{\Phi}_{\mathrm{er}_{V}}(\omega)}{\hat{\Phi} u_{V} r_{V}(\omega)} .
$$

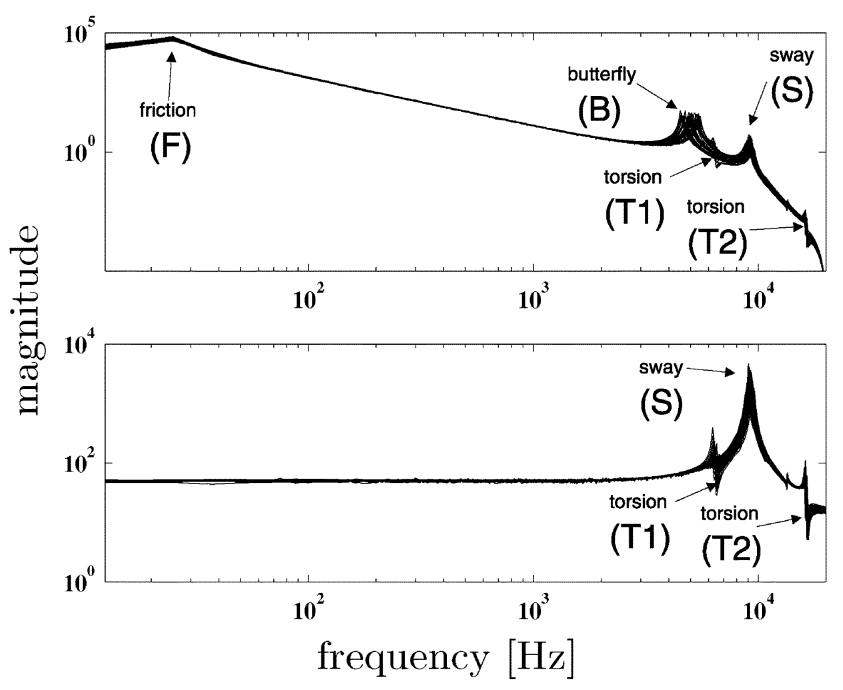

Fig. 2. Amplitude Bode plot of frequency response estimates $\hat{P}_{\mathrm{VCM}}(\omega)$ (top) of the VCM dynamics and $\hat{P}_{\mathrm{MA}}(\omega)$ (bottom) of the MA dynamics.

Since the error rejection function of the VCM control loop can be estimated by

$$
\hat{S}_{\mathrm{VCM}}(\omega)=\frac{\hat{\Phi}_{u_{V} r_{V}}(\omega)}{\hat{\Phi} r_{V} r_{V}(\omega)}
$$

the frequency response of $P_{\mathrm{MA}}$ can be estimated via

$$
\hat{P}_{\mathrm{MA}}(\omega)=\frac{\hat{\Phi}_{\mathrm{er}_{M}}(\omega)}{\hat{\Phi} r_{M} r_{M}(\omega)} \frac{\hat{\Phi}_{r_{V} r_{V}}(\omega)}{\hat{\Phi} u_{V} r_{V}(\omega)} .
$$

To illustrate the variations in the dynamic response of the dualstage actuator, an amplitude Bode plot of estimates $\hat{P}_{\mathrm{VCM}}(\omega)$ and $\hat{P}_{\mathrm{MA}}(\omega)$ of 36 dual-stage actuator systems is given in Fig. 2. Labeled in Fig. 2 are the major structural resonance modes of the dual stage actuator, including the E-block butterfly mode, $(B)$, and the suspension's sway mode, $(S)$, and first and third torsional modes, ( $T 1$ and $T 2)$. Also labeled in Fig. 2 is a lowfrequency resonance mode, $(F)$, which is due to the combined action of nonlinear bearing friction and the ribbon cable.

It can be observed from the estimates $\hat{P}_{\mathrm{VCM}}(\omega)$ that the VCM exhibits significant variations in the E-block butterfly resonance mode $B$, around $5 \mathrm{kHz}$. Variations in the first suspension torsion mode $T 1$ around $6.5 \mathrm{kHz}$ and the sway mode $S$ around $9 \mathrm{kHz}$ can clearly be observed in the estimate $\hat{P}_{\mathrm{MA}}(\omega)$ of the MA.

\section{Dual-Stage Actuator Model Refinement}

A close examination of the frequency responses in Fig. 2 reveals that most of the structural resonance modes in the MA dynamics $P_{\mathrm{MA}}$, and their corresponding variations, are also observed in the VCM dynamics $P_{\mathrm{VCM}}$. It is therefore advantageous from a controller design perspective to define the common structural resonance modes dynamics $P_{\text {ma }}$, which includes the suspension sway $S$ and torsion modes $T 1$ and $T 2$, and can be factorized from both the MA and VCM dynamics, as depicted by the block diagram in Fig. 3. Thus, subsequently we will 


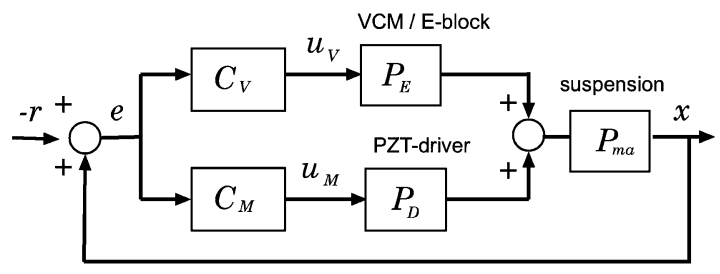

Fig. 3. Dual stage actuator model refinement.

often factorize the dual-stage actuator transfer functions $P_{\mathrm{MA}}$ and $P_{\mathrm{VCM}}$ by the common uncertain dynamics $P_{\mathrm{ma}}$ as follows:

$$
P_{\mathrm{VCM}}=P_{\mathrm{ma}} P_{E} \quad P_{\mathrm{MA}}=P_{\mathrm{ma}} P_{D}
$$

where $P_{E}$ will denote the VCM/E-block dynamics (without the suspension modes), which includes resonance modes $B$ and $F$, while $P_{D}$ denotes the MA's piezoelectric actuator driver dynamics that is not observed in $P_{\mathrm{VCM}}$ and includes a zero.

The variations in the dynamic behavior of the dual-stage actuators may have a large influence on the performance and even stability of high bandwidth dual-stage servo systems, since only one servo controller will be used to control several PZT-actuated suspensions in a single hard disk drive. As a result, the dual-stage servo should be designed such that it is robust against these variations to avoid servo performance deterioration.

\section{UNCERTAINTY MODELING}

From the experimental results shown in Fig. 2, it can be observed that a single linear dynamical model $P$ will not capture the dynamical variations of the dual-stage actuator. Moreover, it may be impossible or too costly to model the dynamical behavior of each dual-stage actuator separately for the design of a dual-stage servo controller, as this would require self tuning and monitoring of the dynamic response of the system for each PZT-actuated suspension in each hard disk drive.

Instead of formulating a single dynamical model, a set of models $\mathcal{P}$ can be proposed to account for the variations in the dynamic behavior of the dual-stage actuators. The set of models $\mathcal{P}$ needs to be constructed such that all measured frequency responses can be represented in the set $\mathcal{P}$. In case this set of models $\mathcal{P}$ is constructed in conformance with robust control design techniques, a robust dual-stage servo controller can be designed and analyzed to account for the variations in the dynamical behavior in the dual-stage suspensions. In this paper, we will briefly describe both dynamic and parametric robust uncertainty modeling, and their use in robust controller design synthesis.

\section{A. Modeling Dynamic Unstructured Uncertainty}

For the experimental data of the dual-stage actuator shown in Fig. 2, variations in the dynamic behavior that have an unknown cause or structure can easily be represented by a multiplicative dynamic uncertainty description

$$
\mathcal{P}_{m}=\left\{P \mid P=P_{0}\left(1+W_{m} \Delta_{m}\right),\left\|\Delta_{m}\right\|_{\infty} \leq 1\right\}
$$

that is well suited for representing unstructured dynamical variations [8], [9]. In (2), $P_{0}$ is used to denote the nominal model, and $W_{m}$ is a weighting function, which models the size and shape of the multiplicative perturbation.
The stable (and possibly structured) norm bounded dynamic perturbation $\Delta_{m}$ is a bounded perturbation that models the dynamic variations in the frequency response measurements. To complete the multiplicative unstructured uncertainty description, both the nominal model $P_{0}$ and a stable and stably invertible weighting function $W_{m}$ in (2) need to be derived from the experimental data.

\section{B. Modeling Parametric Structured Uncertainty}

More detailed information on structural variations in the location and damping of the resonance modes of the dual-stage actuator should be captured using a structured uncertainty description. Assuming uncertainty on stiffness and damping parameters, it is possible to describe variations of the MA and the VCM dynamics as transfer functions that include series combinations of second order uncertain modal transfer functions, with parameters that are affine functions of constant perturbations. In continuous time, such transfer functions can be expressed as

$$
P(s)=P_{c}(s) P_{s}(s)
$$

where $P_{c}(s)$ contains the modes of the plant dynamics that can be modeled with certainty and $P_{s}(s)$ contains the uncertain modes of the plant dynamics, which will be modeled by structured parametric uncertainties. In our case, since most of the uncertainty involves resonance modes, $P_{s}(s)$ can be factorized by a product of second order transfer functions with uncertain coefficients

$$
P^{s}(s)=\prod_{j=1}^{m} \frac{b_{0}^{j} s^{2}+b_{1}^{j} s+b_{2}^{j}}{s^{2}+a_{1}^{j} s+a_{2}^{j}}
$$

where $m$ is the total number of uncertain modes, and the superscript $j$ is used to index each uncertain mode. The uncertain coefficients in (4) are assumed to be affine functions of constant but unknown perturbations

$$
\begin{array}{ll}
b_{i}^{j}=b_{i o}^{j}\left(1+M_{b i}^{j} \delta_{b i}^{j}\right) & i=1,2 \\
a_{i}^{j}=b_{i o}^{j}\left(1+M_{a i}^{j} \delta_{a i}^{j}\right) & i=1,2
\end{array}
$$

where $\left\{b_{i o}^{i}, a_{i o}^{i}, i=1,2\right\}$ are the nominal values of the coefficients, $\left\{M_{b i}^{j}, M_{a i}^{j}, i=1,2\right\}$ are constant weighting coefficients, and $\left\{\delta_{b i}^{j}, \delta_{a i}^{i}, i=1,2\right\}$ are structured perturbations of unity magnitude. The set of structured model variations is then given by

$$
\begin{aligned}
\mathcal{P}_{s} & =\left\{P_{c}(s) P_{s}(s),\left|\delta_{b i}^{i}\right| \leq 1,\left|\delta_{a i}^{j}\right| \leq 1\right\} \\
j & =1, \ldots, m, i=1,2
\end{aligned}
$$

where $P_{s}(s)$ is given by (4) and (5). To complete the structured uncertainty description, both the nominal coefficients $\left\{b_{i o}^{i}, a_{i o}^{j}, i=1,2\right\}$ and the weighting coefficients $\left\{M_{b i}^{j}, M_{a i}^{j}, i=1,2\right\}$ need to be identified from the experimental data.

It should be noted that, if necessary, the unstructured uncertainty model set $\mathcal{P}_{m}$ in (2) and the structured uncertainty model set $\mathcal{P}_{s}$ in (6) can be combined. In this way a single linear fractional transformation (LFT) based uncertainty model set $\mathcal{P}$ 
can capture both structured and unstructured modeling uncertainty. In the next two section we illustrate how both dynamic unstructured and parametric structured uncertainty models for dual-stage actuators can be derived on the basis of experimental frequency domain data.

\section{DYNAMIC UNCERTAINTY MODELING OF DUAL-STAGE ACTUATORS}

\section{A. Characterization of the Nominal Frequency Response}

Let $F_{k}(\omega) \in \mathbb{C}$ represent the $k$ th complex frequency response function (FRF) measured at a finite number of frequency points $\omega \in \Omega . F_{k}(\omega)$ may denote the different measured frequency response measurement $\hat{P}_{\mathrm{vcm}}(\omega)$ or $\hat{P}_{\mathrm{ma}}(\omega)$ and in case of the 36 measurements of the dual- stage actuators displayed in Fig. 2, the index $k$ satisfies $k=1, \ldots, 36$.

The worst case upper bound for a multiplicative model error is given by

$$
\delta_{f}(\omega)=\max _{k=1, \ldots, p}\left\|\frac{F_{k}(\omega)-F_{\text {nom }}(\omega)}{F_{\text {nom }}(\omega)}\right\| \quad \omega \in \Omega
$$

where $F_{\text {nom }}(\omega)$ denotes the nominal FRF to be determined. In order to find a nominal FRF $F_{\text {nom }}(\omega) \in \mathbb{C}$ with the smallest multiplicative error $\delta_{f}(\omega)$, an optimization has to be solved for each $\omega \in \Omega$. By setting $F_{\text {nom }}(\omega)=\alpha(\omega)+i \beta(\omega)$, the minimization of $\delta_{f}(\omega)$ with respect to $F_{\text {nom }}(\omega)$ can be expressed as follows:

$$
\begin{aligned}
\delta_{f}(\omega) & =\min _{\alpha(\omega), \beta(\omega)} \max _{k=1, \ldots, p} J\left(F_{k}, \alpha, \beta\right) \quad \text { for } \omega \in \Omega \\
J\left(F_{k}, \alpha, \beta\right) & =\left\|\frac{F_{k}(\omega)-(\alpha(\omega)+i \beta(\omega))}{\alpha(\omega)+i \beta(\omega)}\right\| .
\end{aligned}
$$

The computation of $\alpha(\omega), \beta(\omega) \in \mathbb{R}$ in (8) can be written as a linear feasibility problem that can be solved via convex optimization for each $\omega \in \Omega$. This guarantees a unique solution at every frequency $\omega \in \Omega$ and it should be noted that the computation of $F_{\text {nom }}(\omega)$ does not resemble the geometric mean $\sum_{k=i}^{p} F_{k}(\omega)$ as the geometric mean does not minimize the worst case multiplicative (or even additive) uncertainty bound $\delta_{f}(\omega)$ in (7).

\section{B. Estimation of Low Order Nominal Model}

As $F_{\text {nom }}(\omega), \omega \in \Omega$ is still in the form of frequency domain data points, a parametric nominal model of preferably low order is needed for the robust $\mathrm{H}_{2}$ controller design synthesis presented in this paper. Due to the inherent discrete-time nature of the hard disk drive servo control algorithm, it is necessary to construct discrete time models.

Low complexity requirements on the nominal model rule out interpolation algorithms [10]-[12], as they tend to give fairly high order models. To address the limited complexity, a discretetime nominal model $P_{0}(s)$ is parameterized in an $n$th transfer function representation

$$
P_{0}(z, \theta):=\frac{b_{0}+b_{1} z-1+\cdots+b_{n} z^{-n}}{1+a_{1} z^{-1}+\cdots+a_{n} z^{-n}} .
$$

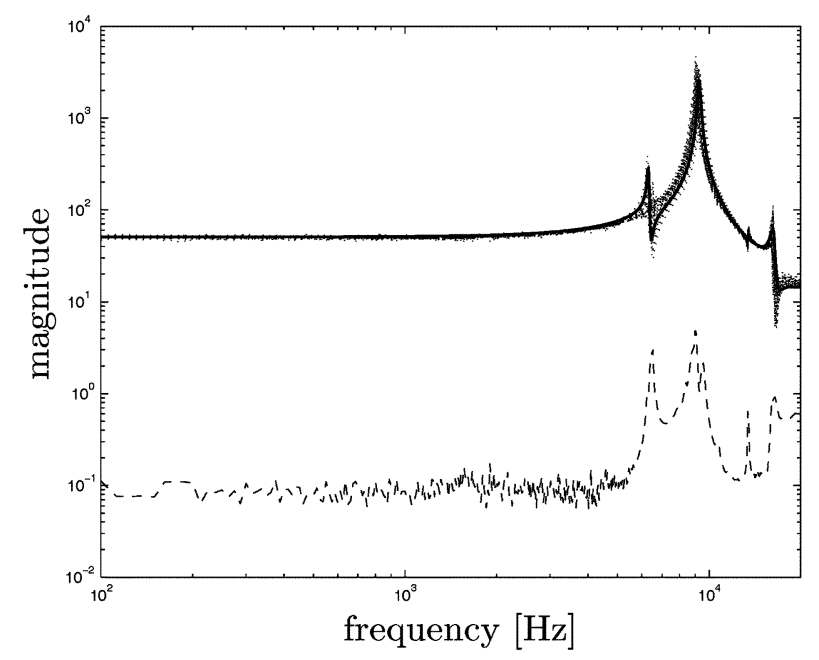

Fig. 4. Frequency measurements $\hat{P}_{\mathrm{MA}}(\omega)$ (dotted) and computed nominal discrete-time sixth order model $P_{0}$ (solid), and resulting multiplicative error bound $\delta_{m}(\omega)$ from (10) (dashed).

The parameter

$$
\theta:=\left[b_{0}, b_{1}, \ldots, b_{n}, a_{1}, \ldots, a_{n}\right]
$$

is found by a curve fit procedure [13] in which the curve fit error

$$
E(\omega, \theta):=\frac{F_{\text {nom }}(\omega)-P_{0}\left(e^{j \omega}, \theta\right)}{F_{\text {nom }}(\omega)} W(\omega), \omega \in \Omega
$$

is minimized.

It should be noted that a (weighted) multiplicative curve fit error is being considered in (9) to approximate the minimization of the upper bound of any unstructured multiplicative uncertainty

$$
\delta_{m}(\omega)=\max _{k}\left\|\frac{F_{k}(\omega)-P_{0}(\omega)}{P_{0}(\omega)}\right\|, \omega \in \Omega .
$$

The additional frequency dependent function $W(\omega)$ can be used to influence the curve fitting of the nominal frequency response data $F_{\text {nom }}(\omega)$. To illustrate the results of the curve fitting procedure, we apply it to the nominal FRF $F_{\text {nom }}(\omega)$ of the MA to estimate a discrete-time nominal model $P_{0}$ of order $n=6$. The Bode plot of this model has been depicted in Fig. 4.

\section{Spectral Over Bounding of Multiplicative Dynamic Uncertainty}

With the given estimated nominal model $P_{0}$, the resulting multiplicative model error bound $\delta_{m}(\omega)$ in (10) needs to be bounded from above by a low order stable and stably invertible discrete time transfer function $W_{m}\left(e^{j \omega}\right)$ such that

$$
\left|W_{m}(j \omega)\right| \geq \delta_{m}(j \omega), \forall \omega \in \Omega .
$$

To address the limited complexity of the weighting function $W_{m}(\omega)$, the weighting function $W_{m}$ can be parameterized in a transfer function representation based on a spectral factorization where

$$
\begin{aligned}
\tilde{W}_{m}(\omega, \tilde{\theta}): & =W_{m}\left(e^{j} \omega, \theta\right) W_{m}^{*}\left(e^{j \omega}, \theta\right) \\
& =\frac{\beta_{0}+\beta_{1} 2 \cos (\omega)+\cdots+\beta_{n} 2 \cos (n \omega)}{1+\alpha_{1} 2 \cos (\omega)+\cdots+\alpha_{n} 2 \cos (n \omega)}
\end{aligned}
$$




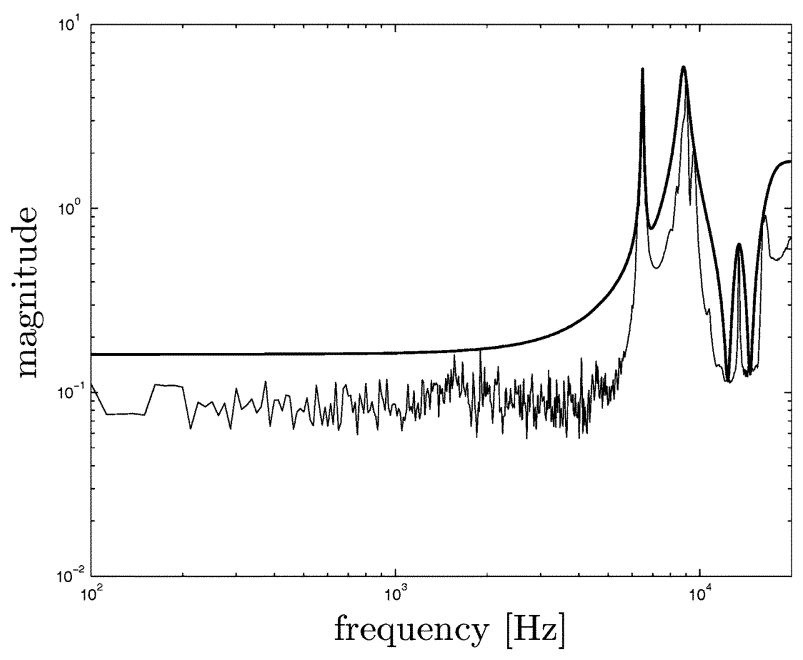

Fig. 5. Amplitude Bode plot of multiplicative error bound $\delta_{m}(\omega)$ from (10) (solid thin line) and stable and stably invertible discrete-time eighth order model $W_{m}\left(e^{j \omega}\right)$ (solid line).

in which $*$ denotes the complex conjugate. In (12) the relation $z^{k}+z^{-k}=e^{j k w}+e^{-j k w}=2 \cos (k \omega), k=1, \ldots, n$ has been used to parametrize $\tilde{W}_{m}(\omega)$. To simplify notations, the sampling time $\Delta T$ of the discrete time filter $W_{m}$ is normalized to $\Delta T=1$. The parameter $\tilde{\theta}$ to be estimated is given by

$$
\tilde{\theta}:=\left[\beta_{0}, \beta_{1}, \ldots, \beta_{n}, \alpha_{1}, \ldots, \alpha_{n}\right]
$$

and the parameter $\theta$ in $W_{m}\left(e^{j \omega}, \theta\right)$ can be found by spectral factorization of $\tilde{W}_{m}(\tilde{\theta})$, provided $\tilde{W}_{m}(\tilde{\theta})>0 \forall \omega$ [14].

The approximation and over-bounding of the frequency dependent multiplicative upper bound $\delta_{m}(\omega)$ by a discrete time parametric model $W_{m}\left(e^{j \omega}\right)$ is addressed by formulating a spectral over-bounding procedure similar as in [15] or [16]. In the spectral over-bounding procedure, the constrained min-max optimization

$$
\begin{aligned}
& \min _{\tilde{\theta}} \max _{\omega \in \Omega}\left|\left(\delta_{m}^{2}(\omega)-\tilde{W}_{m}(\omega, \tilde{\theta})\right) W(\omega)\right| \\
& \text { subjected to } \\
& \tilde{W}_{m}(\omega, \tilde{\theta}) \geq \delta_{m}^{2}(\omega), \omega \in \Omega \\
& \tilde{W}_{m}(\omega, \tilde{\theta})>0, \forall_{\omega}
\end{aligned}
$$

is solved via linear programming techniques. Similar to (9), a frequency dependent weighting function $W(\omega)$ can be used to emphasize certain frequency areas.

To illustrate the result of the spectral over bounding, an amplitude Bode plot of the estimated stable and stably invertible weighting function $W_{m}\left(e^{j \omega}\right)$ has been depicted in Fig. 5. The model $W_{m}\left(e^{j \omega}\right)$ is an eighth order discrete-time model that over bounds the unstructured multiplicative uncertainty bound $\delta_{m}(\omega)$ for all $\omega \in \Omega$. It can be observed that the model $W_{m}\left(e^{j \omega}\right)$ provides a tight overbound of $\delta_{m}(\omega)$, especially at the sharp transitions in the multiplicative uncertainty.
TABLE I

NOMINAL PARAMETERS AND WEIGHTING COEFFICIENTS FOR THE COMMON SUSPENSION MODES STRUCTURED UNCERTAINTY MODEL $\mathcal{P}_{\text {ma }_{s}}$

\begin{tabular}{|c|ccccc|}
\hline mode $j$ & $b_{0 o}^{j}$ & $b_{10}^{j}$ & $b_{20}^{j}$ & $a_{10}^{j}$ & $a_{2 o}^{j}$ \\
\hline $1(T 1)$ & 1 & 720 & $1.598 \mathrm{e} 9$ & 208.2 & $1.575 \mathrm{e} 9$ \\
$2(S)$ & 0 & 0 & $3.316 \mathrm{e} 9$ & 1015 & $3.316 \mathrm{e} 9$ \\
$3(T 2)$ & 1 & 6300 & $1.079 \mathrm{e} 10$ & 2700 & $1.02 \mathrm{e} 10$ \\
\hline
\end{tabular}

\begin{tabular}{|c|cccc|}
\hline mode $j$ & $M_{b 1}^{j}$ & $M_{b 2}^{j}$ & $M_{a 1}^{j}$ & $M_{a 2}^{j}$ \\
\hline $1(T 1)$ & -0.03 & -0.03 & 0 & 0.05 \\
$2(S)$ & 0 & 0.12 & 0.1 & 0.12 \\
$3(T 2)$ & -0.01 & -0.01 & 0 & 0.05 \\
\hline
\end{tabular}

\section{PARAMETRIC UnCERTAinTy MODELING OF DUAL-STAGE ACTUATORS}

\section{A. MA Structured Uncertainty Modeling}

Variations in dual-stage actuator dynamics can also be captured using a structured uncertainty description by assuming specific variations on stiffness and damping of the dual-stage actuator. Similarly as in a dynamic unstructured uncertainty description, the complexity of the uncertainty model is limited to facilitate the computational complexity of the numerical technique used to determine the controller parameters.

As discussed in Section II-C, the common suspension dynamics $P_{\mathrm{ma}}$ can be factorized from both $P_{\mathrm{MA}}$ and $P_{\mathrm{VCM}}$. In the case of the MA, the PZT-driver dynamics, $P_{D}$ in (1) is known with sufficient certainty, and only the common suspension dynamics $P_{\mathrm{ma}}$ is assumed to be uncertain. The structured uncertainty model set $\mathcal{P}_{E_{s}}$ can be described with $P_{\text {ma }}$ in (1) given by (3) by setting $P_{c}(s)=1$ and setting the number of uncertain resonance modes of $P_{s}(s)$ in (4) to $m=3$. From the measured frequency responses it was observed that any perturbations of the suspension's sway mode $(S)$ and the two torsional modes ( $T 1$ and $T 2$ ), which are labeled in Fig. 2, occur simultaneously. This observation leads to a set defined by (5) in which all uncertain parameters can be modeled by a single perturbation $\delta_{1}$, i.e.,

$$
\delta_{b i}^{j}=\delta_{a i}^{j}=\delta_{1}, j=1,2,3 i=1,2 .
$$

The nominal parameter values and constant weighting coefficients for the parameter set defined by (5) are given in Table I, while the MA PZT-driver dynamics, $P_{D}$ in (1) is given by

$$
P_{D}(s)=4.0026 \cdot 10^{-4} s+47.3151 \text {. }
$$

\section{B. E-Block Structured Uncertainty Modeling}

For the E-block, the structured uncertainty model set $\mathcal{P}_{E_{s}}$ can be described with $\mathcal{P}_{E}$ in (1) given by (3) by setting

$$
P_{c}(s)=\left(\frac{1.1821 \cdot 10^{5}}{s+1.1821 \cdot 10^{5}}\right)
$$

and setting the number of uncertain resonance modes of $P_{s}(s)$ in (4) to $m=2$. In this case we are considering as uncertain dynamics the friction/ribbon cable mode $(F)$ and the E-block's butterfly mode, $(B)$, which are labeled in Fig. 2. The uncertain 
TABLE II

NOMinAl PARAMETERS AND WEIGHTING COEFFICIENTS VALUES FOR THE E-Block Structured UnCERTAinty Model SET $\mathcal{P}_{E_{S}}$

\begin{tabular}{|c|ccccc|}
\hline mode $j$ & $b_{00}^{j}$ & $b_{10}^{j}$ & $b_{20}^{j}$ & $a_{10}^{j}$ & $a_{20}^{j}$ \\
\hline $1(F)$ & 0 & 0 & $4.206 \mathrm{e} 8$ & 51.175 & $2.365 \mathrm{e} 4$ \\
$2(B)$ & 0 & 0 & $1.003 \mathrm{e} 9$ & 569.8 & $1.003 \mathrm{e} 9$ \\
\hline
\end{tabular}

\begin{tabular}{|c|cccc|}
\hline mode $j$ & $M_{b 1}^{j}$ & $M_{b 2}^{j}$ & $M_{a 1}^{j}$ & $M_{a 2}^{j}$ \\
\hline $1(F)$ & 0 & 0.3 & 0 & 0 \\
$2(B)$ & 0 & 0.35 & 0 & 0.35 \\
\hline
\end{tabular}

parameters in the set defined by (5) are perturbed by two perturbations: Uncertainty perturbation $\delta_{2}$ is used to vary the DC gain of the E-block dynamics, while perturbation $\delta_{3}$ is used to vary the butterfly resonance mode $(B)$. Thus

$$
\begin{array}{ll}
\text { Mode 1(F): } & \delta_{b i}^{1}=\delta_{a i}^{1}=\delta_{2}, i=1,2 \\
\text { Mode 2(B): } & \delta_{b i}^{2}=\delta_{a i}^{2}=\delta_{3}, i=1,2 .
\end{array}
$$

The nominal parameter values and constant weighting coefficients for the parameter set defined by (5) are given in Table II.

\section{Discretization and Validation of Parametric Uncertainty Model}

With the procedure outlined in the previous sections, the variations in the location and damping of the resonance modes of the dual-stage actuator are assumed to be captured by a structured uncertainty description that requires only three uncertainty perturbations $\delta_{i}^{\prime} s$. The first uncertainty perturbation, $\delta_{1}$, is used to describe the structured uncertainty model set for the MA dynamics, while $\delta_{2}$ and $\delta_{3}$ uncertainty perturbations are used to describe the structured uncertainty model set of the E-block dynamics.

Since we have three uncertain parameters $\delta_{i}$, there are $2^{3}$ "extreme" cases, denoted by $\Delta_{k}, k=1,2, \ldots, 8$, where each parameter $\delta_{i}$ for $i=1,2,3$ takes the value of -1 or 1 . For each extreme case, the continuous-time uncertainty model can be transformed to a discrete-time model, in which the state space matrices $(A, B)$ are given as follows:

$$
A_{d}^{k}:=e^{A\left(\Delta_{k}\right) T}, B_{d}^{k}:=\int_{0}^{T} e^{A\left(\Delta_{k}\right) \tau} d \tau \cdot B\left(\Delta_{k}\right)
$$

where $T=25 \cdot 10^{-6}$ (s) is a sampling period. It can be noted here that for a robust controller design presented in this paper, the convex combinations of the eight discrete-time $(A, B)$-matrices

$$
\mathcal{P}_{d}:=\left\{P(z)=D+C(z I-A)^{-1} B,[A, B] \in \mathcal{B}\right\}
$$

is used, where

$$
\mathcal{B}:=\left\{[A, B]=\sum_{k=1}^{8} \alpha_{k}\left[A_{d}^{k}, B_{d}^{k}\right], \alpha_{k} \geq 0, \sum_{k=1}^{8} \alpha_{k}=1\right\} .
$$

In order to validate the assumption that the structural variations of the dual-stage actuator can be captured by a (convex combination) of only three uncertainty perturbations $\delta_{i}^{\prime} s$, in
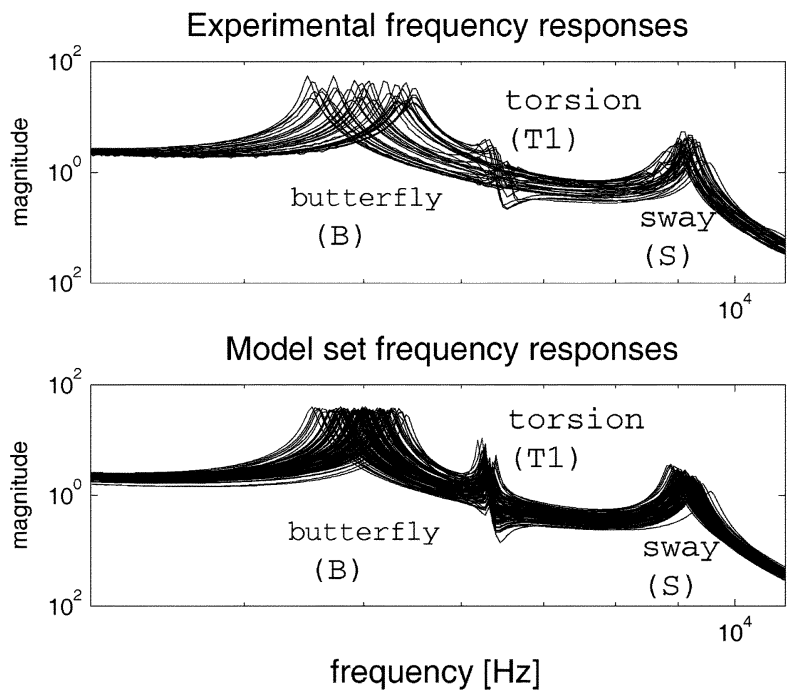

Fig. 6. Comparisons between experimental and simulation frequency responses for $P_{V \text { CM }}$.
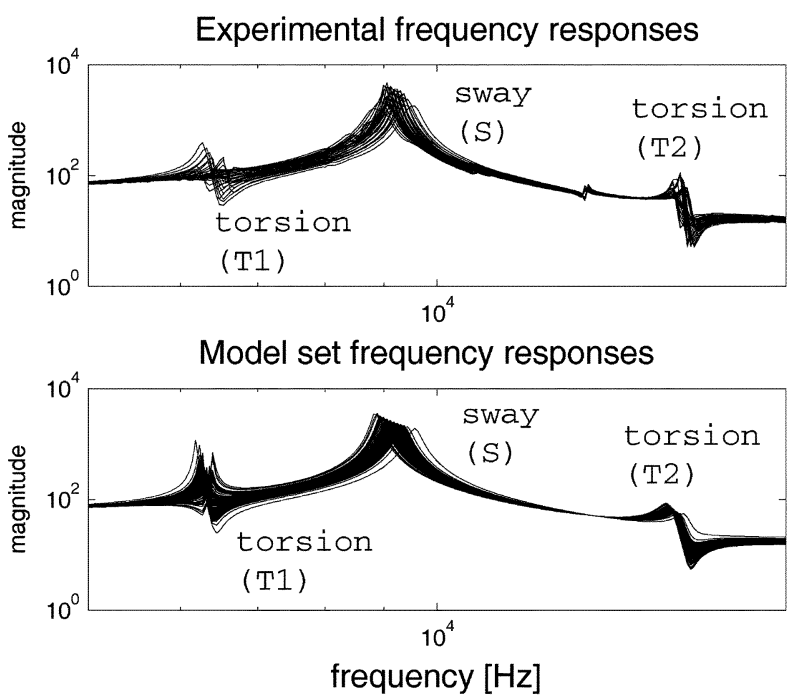

Fig. 7. Comparisons between experimental and simulation frequency responses for $P_{\mathrm{MA}}$.

Fig. 6 and Fig. 7 a comparison is made between the experimental data and randomly picked 100 discrete-time models from the structured model set $\mathcal{P}_{d}$. Both figures show that the structured parametric variations closely resemble the observed dynamic variation in the measured frequency responses.

\section{Robust CONTROL DESIGN}

\section{A. Performance Requirements}

The performance of track-following servo systems is most frequently measured in terms of the variance of the position error signal (PES) $e$ in Fig. 1. Therefore, the most important performance measurement of a servo system is its $H_{2}$ norm, as measured from external stochastic disturbances to the PES.

The uncertainty modeling techniques described in the previous sections can be used in conjunction with modern robust 
control techniques, to design control systems that minimize the $\mathrm{H}_{2}$ norm of the closed loop system under robustness constraints. When the uncertain model set includes dynamic uncertainties, as described in Section IV, mixed $H_{2} / H_{\infty}$ and mixed $H_{2} / \mu$, synthesis techniques can be employed to design the control systems [17]. Moreover, the mixed $H_{2} / \mu$, technique can also handle structured parametric uncertainties. However, these techniques can only guarantee the $H_{2}$ performance of the nominal system and the stability of the control system to the uncertain model set, but not the robust performance of the control system, which may be of critical importance.

In this section, we illustrate the use of the design technique in [18] to design a robust $H_{2}$ control system, when model uncertainty is described by the parametric structured uncertainty set in Section V. Further details regarding the control synthesis methodology are contained in [19].

\section{B. Control System Generalized Plant}

In order to formulate the robust $H_{2}$ control problem, it is necessary to define the nature of the stochastic disturbances to the system and the performance variable, $z$, whose variance will be minimized. In this simplified example, we will only consider as the stochastic disturbance the track runout $r$ and neglect the exogenous signals $r_{V}$ and $r_{M}$ in Fig. 1. We model the runout $r$ as

$$
r=W_{r} w_{r}
$$

where $W_{r}$ is the discrete-time equivalent of the continuous-time shaping filter given by

$$
W_{r}(s)=\frac{3.162 s+1.987 \cdot 10^{5}}{s+628.3}
$$

and $w_{r}$ is discrete-time white noise with unit variance. The runout shaping filter $W_{r}$ can be used as a design tool for shaping the error rejection sensitivity transfer function. The other design tool for $\mathrm{H}_{2}$ based synthesis is the definition of the performance variable $z$. In this simplified example, we define $z$ to be the three-dimensional vector $z=\left[\begin{array}{lll}e & Q_{V} u_{v} & Q_{M} u_{m}\end{array}\right]^{T}$, where the scalars $Q_{V}$ and $Q_{M}$ are control input weights, which are chosen by the designer. In this example, we set $Q_{V}=0.1$ and $Q_{M}=5 \cdot 10^{-5}$.

Utilizing the parametric structure model set described in Section $\mathrm{V}$, the feedback system in Fig. 1 can be rearranged as a connection of a generalized plant $G$, an uncertainty block $\Delta$, and a controller $C$ to be designed. The uncertain system from $\left[w_{r} u\right]^{T}$ to $[z x]^{T}$ can be described as follows:

$$
\left[\begin{array}{c}
z \\
x
\end{array}\right]=\left[D+C(s I-A(\Delta))^{-1} B(\Delta)\right]\left[\begin{array}{c}
w_{r} \\
u
\end{array}\right]
$$

where $A(\Delta)$ and $B(\Delta)$ are affine with respect to $\Delta:=$ $\operatorname{diag}\left[\delta_{1} I, \delta_{2} I, \delta_{3} I\right]$.

\section{Robust $\mathrm{H}_{2}$ Synthesis}

Denoting the transfer function from $w_{r}$ to $z$ by $T_{z w_{r}}(C,[A, B])$, a robust track-following control problem can be formulated as follows: Design a controller $C$ that stabilizes the closed-loop system for all $[A, B] \in \mathcal{B}$, and minimizes the

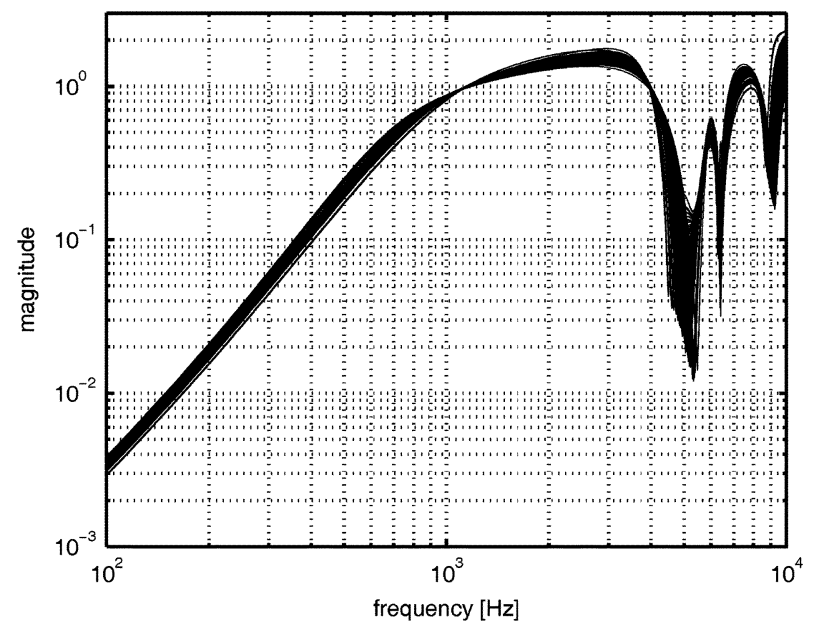

Fig. 8. Amplitude Bode plot of sensitivity functions for sampled models.

worst-case RMS value of $z$ against Gaussian white noise $w_{r}$ for all $[A, B] \in \mathcal{B}$, i.e., solve

$$
\min _{C \in \mathcal{K}(\mathcal{B})} \max _{[A, B] \in \mathcal{B}}\left\|T_{z w_{r}}(C,[A, B])\right\|_{2}
$$

where $\mathcal{K},(\mathcal{B})$ is the set of all stabilizing controllers for any $[A, B] \in \mathcal{B}$. To solve this optimization, we apply the technique based on LMIs in [18]. We remark that the proposed robust $\mathrm{H}_{2}$ synthesis methodology is applicable even for multirate and multi-sensing controller design, which may be of importance in the design of future track-following dual-stage servo systems [19]. To ensure that the optimization (14) is able to find a feasible solution, the parametric variations of the matrices $[A, B] \in \mathcal{B}$ are scaled down initially during the control design. As a result, a controller is designed for a smaller set of parametric uncertainty variations that theoretically cannot guarantee stability robustness for all models $P(z)$ in $\mathcal{P}_{d}$. However, the performance of the resulting controller is analyzed for the original (unsealed) parametric variations of the matrices $[A, B] \in \mathcal{B}$.

The resulting discrete-time controller $C(z)$ is of order 13 with a closed-loop bandwidth of approximately $1.5 \mathrm{kHz}$ and a (conventional) gain and a phase margin of approximately $10 \mathrm{~dB}$ and $20^{\circ}$, respectively. In order to further analyze the robustness properties of the controller, 100 randomly selected models $P(z)$ from the parametric model uncertainty set $\mathcal{P}_{d}$ yields a variation in the error rejection function depicted in Fig. 8. It can be observed from this figure that the error rejection or sensitivity functions do not disperse, even in the face of parameter variations. Due to the robust controller design, so-called "wide notches" are included in the controller frequency response to account for the structural perturbations of the high frequency sway and torsion modes.

\section{REAL-TIME CONTROL IMPLEMENTATION}

To illustrate the effectiveness of the robust control algorithm for reduction of PES variance, the designed robust controller is implemented on TMS320C6713 DSP development board with a Texas Instruments floating point DSP. The same spin stand with a 7200 RPM drive and a Polytech LDV position measurement 


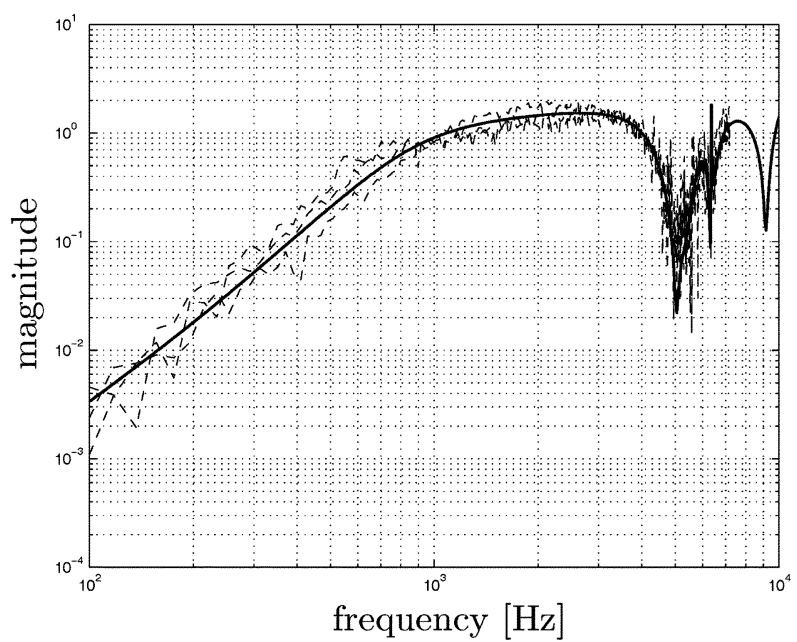

Fig. 9. Amplitude Bode plot of measured error rejection function for three different dual-stage actuators (dashed) and designed nominal error rejection function (solid).

system used during the estimation experiments are exploited for the controller implementation to eliminate any errors due to external interface to the drive.

While keeping the control algorithm the same, the control of three different dual-stage actuators was examined. The three dual-stage actuators were selected on the basis of their observed variations in the dynamical behavior. An estimate of the error rejection function is obtained via spectral analysis and a comparison between the spectral estimate and the designed nominal error rejection function

$$
S=\frac{1}{1+P_{\mathrm{VCM}} C_{\mathrm{vcm}}+P_{\mathrm{MA}} C_{\mathrm{ma}}}
$$

is depicted in Fig. 9. It can be observed that in all three cases, the measured frequency response of the error rejection function closely resembles the frequency response of the error rejection functions obtained in the simulation studies, which are shown in Fig. 8. Any discrepancies are likely due to a (small) computational delay in the DSP implementation.

\section{CONCLUSION}

In this paper, a procedure is presented to characterize an uncertainty model for a dual-stage actuator system in a hard disk drive. Variations in the dynamic response of piezoelectric dual-stage actuators manufactured by Hutchinson Technology Inc. (HIT) are separated in structured and unstructured uncertainties and both are computed via efficient frequency domain based optimization techniques. For characterization of the unstructured uncertainty, the modeling procedure consists of two steps, where first an optimal nominal frequency response is computed and subsequently a low complexity nominal model and dynamic uncertainty weighting function are estimated. For the parametric uncertainty description, it is shown that the variations in dual-stage actuator can be captured with only three parametric variations.
To illustrate the use of an uncertainty model for the design of a servo controller, a robust $H_{2}$ control design method is applied to the parametric uncertainty description to develop a robust high performance servo control algorithm. Real-time implementation of the same control algorithm on several dual-stage actuators validates the robustness of the controller, despite the variations in the dual-stage actuator dynamics.

\section{REFERENCES}

[1] L. Fan, H. Ottesen, T. Reiley, and R. Wood, "Magnetic recording head positioning at very high track densities using a microaactuator-based, two-stage servo system," IEEE Trans. Ind. Electron., vol. 42, no. 3, pp. 222-223, Jun. 1995.

[2] Y. Li and R. Horowitz, "Design and testing of track-following controllers for dual-stage servo systems with pzt actuated suspensions," Microsyst. Technol., vol. 8, pp. 194-205, 2002.

[3] Y. Li, R. Horowitz, and R. Evans, "Vibration control of a PZT actuated suspension dual-stage servo system using a PZT sensor," IEEE Trans. Magn., vol. 39, no. 2, pp. 932-937, Mar. 2003.

[4] A. Teerjuis, S. Cools, and R. de Callafon, "Reduction of flow induced suspension vibrations in a hard disk drive by dual-stage suspension control," IEEE Trans. Magn., vol. 39, no. 5, pp. 2237-2239, Sep. 2003.

[5] R. de Callafon, D. Harper, R. Skelton, and F. Talke, "Experimental modeling and feedback control of a piezo-based milliactuator," J. Inform. Storage Process. Syst., vol. 1, no. 3, pp. 217-224, 1999.

[6] D. Hernandez, S. Park, R. Horowitz, and A. Packard, "Dual-stage trackfollowing servo design for hard disk drives," in Proc. American Control Conf., San Diego, CA, 1999, pp. 4116-4121.

[7] M. Rotunno and R. de Callafon, "Fixed order $H_{\infty}$ control design for dual-stage hard disk drives," in Proc. 39th IEEE Conf. Decision and Control, vol. 4, Sydney, NSW, Australia, 2000, pp. 3118-3119.

[8] S. Skogestad and I. Postlethwaite, Multivariable Feedback Control. Chichester, U.K.: Wiley, 1996.

[9] K. Zhou and J. Doyle, Essentials of Robust Control. Upper Saddle River, NJ: Prentice-Hall, 1998.

[10] J. Partington, "Robust identification and interpolation in $H_{\infty}$," Int. J.Control, vol. 54, pp. 1281-1290, 1991.

[11] S. Pena and R. Snaizer, "Robust identification with mixed time/frequency experiments: Consistency and interpolation algorithms," in Proc. 34th IEEE Conf.Decision and Control, New Orleans, LA, 1995, pp. 234-236.

[12] J. Chen, J. Farell, C. Nett, and K. Zhou, " $H_{\infty}$ identification of multi-variable systems by tangential interpolation methods," IEEE Trans. Autom. Control, vol. 41, no. 12, pp. 1822-1828, Dec. 1996.

[13] R. de Callafon, D. Roover, and P. Van den Hof, "Multivariable least squares frequency domain identification using polynomial matrix fraction descriptions," in Proc. 35th IEEE Conf. Decision and Control, Kobe, Japan, 1996, pp. 2030-2035.

[14] K. Åström, Introduction to Stochastic Control Theory. New York: Academic, 1970.

[15] R. Scheid, D. Bayard, and Y. Yam, "A linear programming approach to characterizing norm bounded uncertainty from experimental data," in Proc. American Control Conf., Boston, MA, 1991, pp. 1956-1958.

[16] R. Scheid and D. Bayard, "A globally optimal minimax solution for spectral overbounding and factorization," IEEE Trans. Autom. Control, vol. 40, no. 4, pp. 712-716, Apr. 1995.

[17] R. Nagamune, X. Huang, and R. Horowitz, "Multi-rate track-following control with robust stability for a dual-stage multi-sensing servo system in HDDs," in Proc. Joint 44th IEEE Conf. Decision and Control and Eur. Control Conf., Seville, Spain, 2005, pp. 3886-3891.

[18] S. Kanev, C. Scherer, M. Verhaegen, and B. D. Schutter, "Robust outputfeedback controller design via local BMI optimization," Automatica, vol. 40, pp. 1115-1127, 2004.

[19] R. Nagamune, X. Huang, and R. Horowitz, "Robust control synthesis techniques for multirate and multi-sensing track-following servo systems in HDDs," Computer Mechanics Lab., Univ. California, Berkeley, Tech. Rep., 2005.

Manuscript received August 8, 2005 (e-mail: callafon@ucsd.edu). 